\section{Bessere Symptomkontrolle bei NSCLC}

Bei nicht-kleinzelligem Lungenkrebs (NSCLC) mit Adenokarzinomhistologie und positivem EGFR-Mutationsstatus führt Afatinib (Tomtovok ${ }^{\circledR}$ ) zu einer effektiveren Kontrolle der tumorassoziierten Symptome und zu einer höheren Lebensqualität als die Standardbehandlung. Dies geht aus den Daten der LUXLung-3-Studie hervor. Im Vergleich zur bislang als am wirksamsten geltenden Chemotherapie (Pemetrexed plus Cisplatin) waren in der Afatinib-Gruppe die Symptome Husten, Kurzatmigkeit und Schmerzen verbessert. Afatinib verzögerte gegenüber Pemetrexed/Cisplatin auch das Zeitintervall bis zur Verschlechterung von Husten und Kurzatmigkeit. Wichtige Ergebnisse waren weiterhin, dass in der AfatinibGruppe bessere körperliche, kognitive und rollenspezifische Funktionen sowie eine höhere Lebensqualität im Vergleich zur Chemotherapie zu verzeichnen waren. Bereits präsentierte Daten der Studie ergaben, dass die Patienten unter Afatinib fast ein Jahr ohne Tumorprogression überlebten (mediane progressionsfreie Überlebenszeit: 11,1 Monate) - unter Pemetrexed/Cisplatin waren es nur 6,9 Monate.

Nach Informationen von Boehringer Ingelheim

\section{Neue Therapieoption bei AML}

Für Patienten ab 65 Jahren mit neu diagnostizierter AML, bei denen eine Standard-Induktionstherapie nicht infrage kommt, hat die European Medicines Agency nun Decitabin (Dacogen $^{\circledR}$ ) zugelassen. Grundlage für die Entscheidung waren Ergebnisse einer Phase-III-Studie, in der Decitabin mit niedrig dosiertem Cytarabin oder supportiver Therapie verglichen wurde. Dabei führte Decitabin zu besseren Ansprechraten (komplette Remission mit oder ohne vollständige Erholung der Thrombozyten: 18 vs. $8 \%$ ), längerem progressionsfreien Überleben $(3,7$ vs. 2,1 Monate) und längerem Gesamtüberleben (7,7 vs. 5,0 Monate).

Nach Informationen von Janssen-Cilag

Multiples Myelom

\title{
Dreierkombination mit Bendamustin
}

Bendamustin ist für die Primärtherapie des multiplen Myeloms (MM) in Kombination mit Prednison zugelassen. Kürzlich präsentierte Daten zeigen, dass es auch mit Thalidomid, Lenalidomid oder Bortezomib kombiniert werden kann.

So wurde bei stark vorbehandelten therapierefraktären oder rezidivierten Patienten mit Bendamustin in Kombination mit Thalidomid und Prednison eine Ansprechrate von bis zu $86 \%$ und ein medianes progressionsfreies Überleben von 11 Monaten erreicht [Pönisch W et al. Br J Haematol. 2008;143(2):191-200]. Ähnlich gute Ergebnisse wurden mit der Kombination von Bendamustin mit Lenalidomid und Prednison erreicht [Pönisch W et al. EHA 2012; abstr. 848].

Ein Aspekt, der nach Wolfram Pönisch, Leipzig, besonders wichtig ist: Bendamustin kann auch bei niereninsuffizienten $\mathrm{Pa}$ - tienten und dialysepflichtigen Patienten eingesetzt werden. Eine retrospektive Analyse hatte ergeben, dass auch diese $\mathrm{Pa}$ tienten von der Kombination Bendamustin, Bortezomib und Prednison profitieren [Pönisch W et al. Blood. (ASH Annual Meeting) 2011;118: Abstr. 2938].

Doris Berger

Satellitensymposium „Lebensqualität sichern Multiples Myelom erfolgreich therapieren", anlässlich der Jahrestagung der Deutschen, Österreichischen und Schweizerischen Gesellschaften für Hämatologie und Onkologie, Stuttgart, 21.10.2012; Veranstalter: Mundipharma

\section{Erythropoetin-Biosimilar als Alternative zur Transfusion}

Die Gabe von Erythrozytenkonzentraten kann zu einem erhöhten Rezidivrisiko beitragen. Als Alternative bietet sich ein Erythropoetin-Präparat mit Niedrig-Dosis-Schema an.

Je nach Tumorentität leiden 25-50\% der onkologischen Patienten an einer Anämie, die ihre Lebensqualität schwer beeinträchtigt. Das in der Leber vermehrt produzierte Hepcidin blockiert die enterale Eisenresorption, erläuterte Hartmut Link, Kaiserslautern. Zudem verhindert dieses Protein die Eisenfreisetzung aus dem retikuloendothelialen System.

Studiendaten weisen darauf hin, dass die Gabe von Erythrozytenkonzentraten das Risiko für Tumorrezidive und karzinombedingte Mortalität erhöhen kann. Als Alternative bieten sich Erythropoesestimulierende Agenzien (ESA) an. Bei korrektem Einsatz beeinträchtigen diese Substanzen die Prognose von Tumorpatienten nicht, betonte Link. Patienten sollten allerdings über das bestehende Thromboembolierisiko aufgeklärt werden.
Mit Epoetin theta (Eporatio ${ }^{\star}$ ) steht ein Biosimilar zur Verfügung, das vergleichbare Ansprechraten wie Referenzpräparate erzielt. Als Besonderheit nannte Link das in Studien geprüfte Niedrigdosis-Schema: Die Therapie begann dabei in niedriger Dosierung von 20.000 I.E pro Woche. War der Hämoglobin-Wert nach vier Wochen nicht um mindestens $1 \mathrm{~g} / \mathrm{dl}$ gestiegen, erfolgte eine Dosisverdopplung, nach weiteren vier Wochen ggf. eine weitere Dosissteigerung um 20.000 I.E. Bereits $52 \%$ der Patienten sprachen auf die niedrigste Dosis an. Der Eisenbedarf unter einer ESA-Therapie ist groß, so Link. Die Blutbildung lässt sich jedoch durch eine zusätzliche intravenöse Eisensubstitution optimieren.

Katharina Arnheim

Satellitensymposium „Wirksamkeit in der Onkologie - Neue Wege gehen" anlässlich der Jahrestagung der Deutschen, Österreichischen und Schweizerischen Gesellschaften für Hämatologie und Onkologie, Stuttgart, 19. Oktober 2012; Veranstalter: Teva 